\title{
Lenguaje: Algo más que un mecanismo para la comunicación
}

\section{Language: More than a Mechanism for Communication}

\author{
Luis Alfredo Miranda Calderón ${ }^{l}$ \\ División de Educación Básica \\ Centro de Investigación y Docencia en Educación (CIDE) \\ Universidad Nacional de Costa Rica \\ Heredia, Costa Rica \\ alfremira@gmail.com
}

Recibido 04 de agosto de 2010 • Aceptado 31 de agosto de 2010

Resumen. El propósito de este artículo es reflexionar acerca de la importancia del lenguaje en el proceso de aprendizaje de las personas, en contraste con la visión tradicional con la que se ha orientado la enseñanza de la lengua y su incidencia en el campo educativo. Para ello, se hace un análisis desde distintas perspectivas y visiones que aportan diversos estudiosos, quienes han abordado el tema del lenguaje, sus implicancias en el aprendizaje escolar y en el que realizan los seres humanos a lo largo de su existencia. Ante este contexto, el artículo plantea la necesidad de establecer mecanismos pedagógicos que aborden una nueva visión de la enseñanza de la lengua en cualquiera de sus áreas, visualizando el lenguaje como herramienta para el aprendizaje y como vehículo para alcanzar la expresión integral en todas las modalidades de la educación.

Palabras clave. Lenguaje, lenguaje integral, aprendizaje, enseñanza del español, comunicación.

Abstract. The objective of this paper is to study the importance of language in the learning process of individuals, in contrast to the traditional view that has predominated in the teaching of language and its incidence in the educational field. An analysis is performed from the different perspectives of experts who have studied the language, its learning implications and language development of individuals throughout their lives. This paper brings up the need to establish pedagogical mechanisms to approach a new vision in the teaching of language in any area, considering language as a tool for learning and as a way to reach full expression in all forms of education.

Keywords. Language, integral language, learning, teaching of Spanish, communication.

\footnotetext{
Bachiller en Artes Dramáticas, Bachiller en Ciencias de la Educación en I y II Ciclos, Licenciado en Educación Rural, Magíster con especialidades en Administración Educativa y en Formación de Formadores de la Educación Básica. Maestro rural unidocente durante años en una comunidad rural costera, donde ha realizado diversas investigaciones y exploraciones relacionadas con el aprendizaje mediado por uso y aprovechamiento del entorno natural. Maestro en la Escuela Nueva Laboratorio de la Universidad de Costa Rica, lugar en el que puso en práctica y sistematizó procesos pedagógicos mediados por el teatro como una herramienta para el aprendizaje significativo. Desde 2005 se ha desempeñado como docente en la Universidad de Costa Rica y como académico investigador en la División de Educación Rural de la Universidad Nacional. Actualmente labora como académico en la División de Educación Básica de la Universidad Nacional. Asimismo conforma, en la División de Educación Rural, la comisión responsable del Diseño de la Licenciatura en Educación Secundaria Rural.
} 
El lenguaje ha de ser matemático, geométrico, escultórico. La idea ha de encajar exactamente en la frase, tan exactamente que no pueda quitarse nada de la frase sin quitar eso mismo de la idea.

José Martí

El lenguaje, como elaboración y síntesis del conocimiento humano, constituye quizá la mejor evidencia del paso de nuestra especie por el planeta, a lo largo del tiempo y el espacio. Como

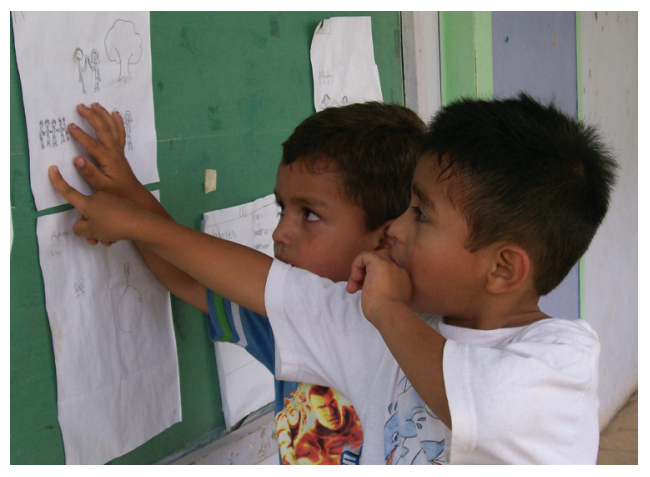
producto indiscutible de los seres humanos, el lenguaje que hemos instrumentalizado ha sido desde siempre un indicador fiel del comportamiento de la sociedad a través el tiempo. Por medio de este podemos vislumbrar el sutil entramado de la sociedad y sus efectos directos e indirectos en el pensamiento y la conciencia colectiva.

El lenguaje, al concebirse como vehículo transmisor del complejo sistema de los valores y de las ideas hegemónicas de cada época y cada sociedad, ha sido -y posiblemente se constituirá en tal- un elemento sustancial y permanente de la evolución social en el devenir del tiempo. Desde esta visión resulta indispensable analizar de qué forma el lenguaje incide en el pensamiento y viceversa. Cuáles son sus implicaciones en el aprendizaje y de qué manera la pedagogía contemporánea debe considerar dichas nociones para que oriente la concepción de nuevos modelos pedagógicos más acordes con las nuevas tendencias que proclaman una visión integral en la construcción y generación de conocimiento.

Mediante el lenguaje nos aproximamos a nosotros mismos y a los demás, reflexionamos sobre nuestro proceder, nuestras emociones y condiciones internas; a través de lenguaje comprendemos e interpretamos la naturaleza, el mundo de las estructuras físicas y formales; mediante el lenguaje heredamos conocimiento de las generaciones anteriores y trasmitimos ideas a las próximas, construimos imágenes de nuestro ser y el de los otros, elaboramos máquinas, artefactos y programas, pero, lo más fascinante, con el lenguaje indagamos sobre el mismo lenguaje y en su relación intrínseca con el pensamiento.

Los seres humanos nos encontramos ante una paradójica situación en la que somos usuarios y constructores del legado más preciso y contundente de nuestra evolución: el lenguaje.Este encierra el pasado y presente de cualquier cultura o sector de la sociedad humana y representa uno de los mecanismos más brillantes y valiosos ideados e intuidos por nuestra civilización, como bien señala Olson (1999):

(...) el lenguaje es un recurso cultural, y sus implicaciones tienen que desplegarse en un tiempo histórico. La escritura puede permitir la enumeración de las leyes de la cultura, por ejemplo, pero enseñar a alguien a leer y a escribir no mejorará inmediatamente su capacidad para establecer códigos legales. Es necesaria la intervención de un proceso histórico. (...) La educación, tal como la conocemos, es una institución letrada. (p. 62-63)

El lenguaje visto como una actividad intrínseca del aprendizaje constituye el vínculo intuitivo y racional, orgánico y perceptible de nuestra interacción con el entorno físico y social, lo que nos 
conduce a reflexionar cómo es que abordamos su aprendizaje, o mejor dicho, su potencialidad en el campo educativo y sus implicaciones en la pedagogía contemporánea. Alrededor de esta temática se han generado diversas discusiones acerca de cuál es el verdadero papel que los sistemas educativos deben conceder al lenguaje como componente indispensable en la formación integral de la persona.

\section{Visión reduccionista del lenguaje en el currículo escolar}

En el caso de la educación primaria, todavía hoy es común encontrar dificultades para identificar cuál es la verdadera función de la mal denominada área del "Español”. Limitar su constitución como un campo disciplinar y no como una herramienta o instrumento para el aprendizaje y la construcción de conocimiento ha generado un problema de confusión. Esa confusión se debe a la imprecisión histórica en la construcción de este objeto de estudio de la comunicación, concebido más como un conjunto de conocimientos

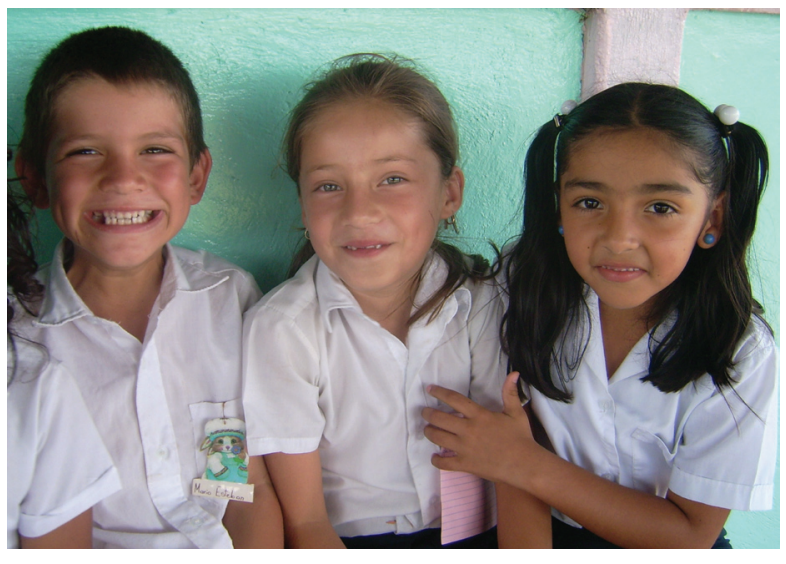
técnicos de una lengua específica que como un medio. Al respecto, Goodman (2008) afirma:

(...) Muchas prácticas escolares tradicionales parecen haber impedido u obstaculizado el desarrollo del lenguaje. En nuestro fervor por hacerlo más fácil, lo hemos hecho difícil. ¿Cómo? Principalmente al dividir el lenguaje natural en fragmentos de tamaño de bocadillo, pequeños pero abstractos (...). (p. 108)

Es más común referirse a la lengua, indistintamente, como un proceso de enseñanza de normas ortográficas y gramaticales, que como pedagogía para la expresión y comunicación integral de la persona. Esto provoca dificultades adicionales, porque no hay referentes teóricos claros o determinados al respecto.

En nuestros días, nociones "difusas" y más bien normativas caracterizan el objeto de estudio del lenguaje. Estas confusiones limitan el progreso racional y la socialización en el campo del lenguaje, lo que constituye obstáculos históricos adicionales para la configuración del aprendizaje como disciplina científica. Por lo tanto aprender a leer y escribir es condición necesaria pero no es suficiente para pertenecer a la cultura escrita, ya que se requiere además de habilidades metacognitivas, cognitivas y afectivas para hacer uso consciente y funcional del lenguaje, ya que interpretar lo que se lee y tener una intención particular cuando se escribe trascienden la capacidad de decodificar signos e interpretar textos en forma literal. El reconocimiento de esos requerimientos llevó a la invención de una institución capaz de brindar a las personas de la "era de la imprenta" la formación necesaria para emplear de manera apropiada recursos culturales sin precedentes.

Esta visión reduccionista del aprendizaje de la lengua como asignatura inerte propicia una serie de imaginarios en torno a ser buen o mal estudiante en Español. Si los niños y niñas no dominan las técnicas o requerimientos propios de esta disciplina, no solo se les condena al fracaso escolar sino que se les cierra una puerta de acceso al conocimiento de otras áreas del currículo, como señala Goodman (2008): 
(...) Desde el punto de vista de los alumnos es un currículum único que enfoca lo que se aprende y que toma en consideración el objetivo que tiende al uso del lenguaje. Pero para el maestro hay siempre una doble tarea: por un lado, la de ofrecer el máximo de oportunidades a los alumnos para que participen en hechos auténticos de habla y de lectoescritura y, por el otro, deben investigar la comunidad, estudiar un tema literario, llevar a cabo un estudio científico sobre ratones o familiarizarlos con quebrados y decimales (...). (p. 119)

Lo anterior nos conduce a comprender que el fortalecimiento y enriquecimiento del lenguaje debe entenderse como una inversión y compromiso de la escuela primaria a corto, mediano y largo plazo; ya que el esfuerzo y el tiempo dedicados a comprender los conceptos propios del lenguaje universal y su estrecha relación con lo que aprendemos nos puede conducir a una educación más justa y equitativa para quienes aprenden. Ellis (1994) señala:

Nada parece más razonable que suponer que la función primordial del lenguaje es la comunicación. Pero al hacer esta suposición caemos de inmediato en una trampa sutil: sin duda un acto particular del uso del lenguaje puede derivar en comunicación entre personas, pero mucho tuvo que haber acontecido antes de que pudieran haber llegado tan lejos.... El lenguaje tiene primero que haber tenido algo que ver con aquello que había por comunicar, y con todo aquello que podría ser considerado como comunicación. No es un mero medio para transmitir información, es también, y más importantemente, el lugar del proceso de decisión de lo que debe ser la información, y de instituir los tipos de información que estarán disponibles para la comunicación. (p. 17)

Pese a lo evidente de esa necesidad, es una tarea pendiente en las escuelas y facultades de educación de las universidades públicas del país, tanto a nivel interno como en el sistema de educación superior pública. La ruptura con un modelo reduccionista del aprendizaje de la lengua se hace impostergable, ya que se ha legitimado con una visión, desde la formación de formadores, difusa, fragmentada, descontextualizada, reproductora del mismo patrón en todos los niveles de la educación. Según Arellano (2002):

El acceso al currículo está mediatizado por el lenguaje, sea oral o escrito. Los conocimientos de las distintas disciplinas se articulan, en gran parte, de forma lingüística y simbólica. Gracias a que el acervo disciplinar se ha conservado en libros y textos escritos, existe una continuidad y una evolución en las ciencias, las técnicas, las artes y las humanidades con múltiples formas de lenguaje. Acceder a estos saberes exige el dominio de ciertas técnicas de escucha, expresión escrita y oral y lectura comprensiva que deben ser practicados de modo habitual en el aula. (p. 21)

Con esta premisa, el lenguaje, en toda su dimensión, debería ser estimulado en todas las experiencias de aula, sin importar el área disciplinar, por lo que debe constituirse en herramienta para acceder a información útil, interactuar con su entorno social y generar conocimiento aplicable y útil. Esta perspectiva nos conduce a la consolidación de la clara idea de que cada individuo aprende de forma única y desarrolla una relación singular, exclusiva e irrepetible con el conocimiento, permeada de sus representaciones, vivencias y de su interacción con su espacio físico y social, lo que nos hace reflexionar acerca de la trascendencia del lenguaje y las formas de comunicación que utilizamos para relacionarnos con el entorno. 
Lo anterior nos puede ayudar a reafirmar, una vez más, la presencia e influencia de nuestras representaciones, no solo en la cotidianidad de cada persona, sino además su fuerte predominio en la vida social, lo que analógicamente es comparable con la noción del cerebro social como lo plantea Ratey (2003):

El cerebro constituye un ejemplo clásico del increíble ingenio de la evolución, que nos hace ser criaturas con mucha capacidad de adaptación... en los mismos principios del su desarrollo, el cerebro es un órgano social: donde si no hay conexión no hay vida. (p. 39)

Lo precedente nos lleva a la conclusión de que el cerebro social colabora en la capacidad del individuo en desarrollar y fijar la interacción social y desempeñarse en su medio de forma dinámica y con múltiples interrelaciones entre sus conocimientos pre-existentes. Esto, sin duda nos conduce, inevitablemente, a una profunda y seria reflexión acerca de: ¿qué es lo que sucede en nuestro cerebro durante el aprendizaje? ¿Cómo aprenden las personas?

\section{Los nuevos aportes de la Neurociencia: itinerario para una renovada concepción del lenguaje}

Las incertidumbres han venido sugiriendo, desde hace décadas y desde los diversos campos que atienden el aprendizaje, la necesidad de estructurar nuevas teorías, enfoques y paradigmas que orienten las prácticas pedagógicas a la luz de los nuevos tiempos e incidan en ellas, desde una visión más científica y real, tal como la psicología, la epistemología en la filosofía y, recientemente, la neurociencia. En el caso de la gestación y adquisición de formas de lenguaje, es indiscutible la evidente relación entre los mecanismos que utilizamos para comunicarnos y sus implicancias en el pensamiento.

Durante décadas, el intento por la comprender la mente, el pensamiento, el lenguaje y el aprendizaje, ha representado una ardua búsqueda, debido en parte a la falta de herramientas interdisciplinarias poderosas para la investigación y la generación de conocimiento actualizado que respalde decisiones educativas. Sin embargo, el mundo de hoy nos sitúa ante una extraordinaria producción de estudios científicos sobre la mente, el cerebro y el lenguaje; sobre los procesos de pensamiento y su influencia en el aprendizaje, y sobre la vasta gama de procesos neuronales que ocurren durante el pensamiento y el aprendizaje. Esta dinámica revolución, ocurrida en el estudio de la mente durante las últimas cuatro o cinco últimas décadas, tiene importantes implicaciones en la pedagogía contemporánea y, consecuentemente, en la formación de los futuros pedagogos.

Hoy en día y, gracias a los aportes de recientes estudios neurológicos, sabemos que obedecemos a estímulos internos y externos, debido a nuestra condición humana, lo que nos lleva a cuestionar cuáles son estos estímulos y de qué forma intervienen en el lenguaje, en el aprendizaje y en la génesis de conocimiento de cada individuo.

El trabajo científico ha abarcado una amplia gama de temas de la cognición y la neurociencia en el aprendizaje, la memoria, el lenguaje y el desarrollo cognitivo. A medida que los científicos continúan estudiando el aprendizaje, emergen nuevos lineamientos, paradigmas y metodologías de investigación, que probablemente modifiquen las concepciones teóricas que, del aprendizaje, se tienen actualmente. Esto debido a que en los últimos años ha aumentado, notablemente, el interés de la neurociencia por estudiar la capacidad cognitiva en todas las edades de los seres humanos. 
Numerosos estudios han demostrado que las aseveraciones sobre las etapas del desarrollo humano, establecidas en el pasado, no son tan universales como cabría esperarse, por lo que se estima que el conocimiento alcanzado en relación con nuestras capacidades cognitivas constituye un conocimiento inacabado en continua transformación, gracias a la incursión de dichos estudios como señala Ratey (2003) al advertir:

Aprendemos a lo largo de la vida adulta ya que nuestros cerebros son maravillosamente plásticos...nuestra estructura cerebral no está predeterminada o fijada. Podemos alterar el desarrollo en marcha de nuestros cerebros, nuestro lenguaje y por lo tanto, nuestras capacidades. (p. 34)

Esta afirmación revalida la idea de precisar el aprendizaje como un fenómeno en el que se fusionan múltiples procesos mentales, entre ellos el lenguaje, el cual constituye el conjunto de procesos dados en todo momento de interacción del individuo con la realidad circundante. En la interacción de las personas con su entorno social se generan aprendizajes en diversos matices que van desde el aprendizaje espontáneo, al aprendizaje estimulado, tal es el caso del aprendizaje gestado en el entorno escolar, gracias a las diversas formas de lenguaje que vehiculizan la comunicación.

Todo lo que emprendemos compromete nuestro pensamiento mediante la forma en que nos comunicamos y "lenguajeamos" aún con nosotros mismos, como enfatiza Goodman (2008):

Los procesos de hablar, escuchar, escribir y leer tienen lugar en el contexto de las exploraciones del mundo: las cosas, los hechos, las ideas y las experiencias. El contenido del currículum toma en cuenta los intereses y las experiencias que los niños tienen fuera de la escuela y, de este modo, incorpora toda la gama de las funciones lingüísticas orales y escritas. Se convierte en un currículum amplio y rico que comienza con el lenguaje y el conocimiento que el alumno posee y construye acerca de ellos. La meta es el crecimiento individual y no el logro de determinados niveles. (p. 119)

Desde esta perspectiva, los aportes de la teoría del aprendizaje de Vigotsky, producto de su explicación del pensamiento y el lenguaje, constituyen un marco explicativo privilegiado para el campo de la educación, preocupada por responder a la pregunta: ¿cómo es posible el aprendizaje? Si precisamos a ciencia cierta cómo aprenden las personas, es posible construir un marco explicativo sobre cómo aprovechar y utilizar el lenguaje para generar la construcción de conocimiento, de manera tal que quien viva la experiencia de la educación alcance conocimientos y desarrolle actitudes y habilidades comunicativas que le van a ser útiles para su desarrollo como individuo, en un entorno social particular. Al respecto Goodman (2008) señala:

Los niños aprenden el lenguaje oral en sus hogares sin que nadie lo divida en fragmentos pequeños. Lo aprenden cuando lo necesitan para expresarse y entienden lo que dicen los otros, siempre que estén con personas que utilicen el lenguaje con sentido y con un propósito determinado. (p. 108)

Una de las consecuencias de la teoría de Vigotsky respecto de la educación es la comunicación y su especificidad. Es decir, no es lo mismo comunicarse socialmente, intrasíquicamente o pedagógicamente. La mediación comunicativa y social de la enseñanza tiene sus propias 
características estructurales y funcionales, que son las que le confieren el carácter de "situación pedagógica", donde se proponen y llevan a cabo acciones de enseñanza y de aprendizaje concretas. Ello supone diferencias fundamentales entre los marcos explicativos del fenómeno pedagógico de las corrientes transmisionista y conductista. Al respecto Olson (1999) advierte:

Pese a que la escuela surgió en la Modernidad como una institución letrada, con las funciones estratégicas de la alfabetización y el desarrollo de los conocimientos y destrezas necesarios para el empleo del lenguaje impreso, en sus inicios no se contó con una definición del objeto de estudio de la Educación ni de los problemas a resolver para que las personas adquieran las competencias necesarias para hacer un uso apropiado de los recursos culturales disponibles en una sociedad basada en textos escritos. (p. 49)

La escuela primaria como institución social encargada de realizar el proceso de alfabetización y formación para la utilización de la lectura y escritura, con el propósito de ponerla al servicio de la producción y reproducción del sistema social, tuvo, además de la alfabetización, la tarea de poner los recursos de la escritura al servicio de la cultura escrita, como lo menciona Olson al referirse que la alfabetización:

(...) no es sólo un conjunto básico de habilidades mentales aisladas de todo lo demás. Es la competencia para explotar un conjunto determinado de recursos culturales. Es la evolución de esos recursos, en conjunción con el conocimiento y la habilidad para explotarlos con fines determinados, lo que constituye la cultura escrita. (p. 65)

El papel que juegan docentes y aprendices en una "situación de enseñanza", desde la teoría sociocultural, es radicalmente distinto a los que anterior y tradicionalmente han marcado la historia de la educación, ya que esta teoría enfatiza la importancia del "comunicarse" consigo (reflexión) y con otros (socialización) para la concreción del aprendizaje significativo.

Si observamos las características de los roles de docentes y estudiantes en los enfoques tradicionales y en el de Vigotsky, es evidente que las habilidades cognitivas que se priorizan son muy distintas. La memoria es la habilidad cognitiva más importante en los tradicionales, mientras que en el de Vigotsky la memoria es importante, pero no es la habilidad cognitiva que se prioriza, sino que ese lugar lo ocupan aquellas habilidades cognitivas que favorecen la metacognición; es decir, el lenguaje como mecanismo para transacción social del conocimiento y la reflexión.

El lenguaje constituye la llave para detonar un proceso inherente al ser humano, que se da a lo largo de la vida: el aprender. Por lo tanto, no requiere necesariamente de la intervención "intencionada" de otra persona para que ocurra, siempre estamos aprendiendo, con o sin intención de hacerlo.

Al interactuar con nuestro entorno estamos descubriendo, elaborando y estableciendo múltiples formas de lenguaje con todos los elementos, aprendiendo de ellos. Este sentido del aprendizaje continuo se ha ignorado, ya que se asume, por defecto, que el aprender solo se da en entornos escolares, con el agravante de la existencia de un rezago significativo, si consideramos el avance de otras actividades del quehacer humano, tal es el caso de la enseñanza formal. En el caso de la pedagogía contemporánea es necesario comprender y consensar que el lenguaje articulado al aprendizaje de todo individuo es continuo y permanente, y que la vida y la mente del niño y la niña desde la pedagogía, así lo requieren. 

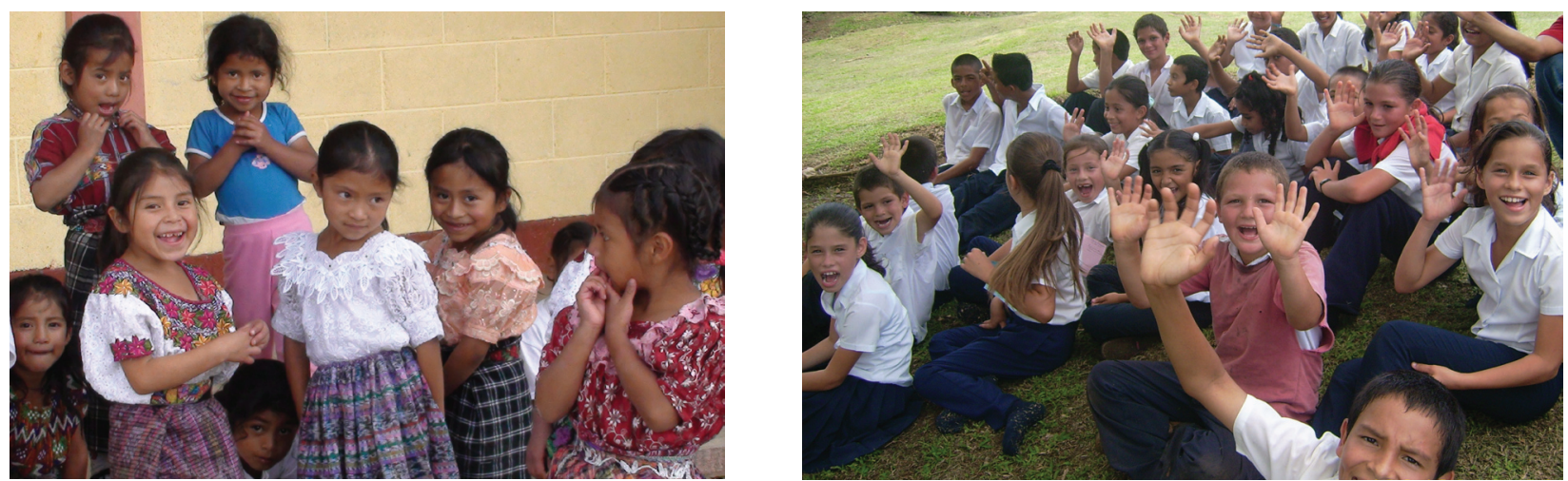

\section{Me atrevo a concluir...}

Quizá, el sitio ideal para observar y analizar el fantástico fenómeno del aprendizaje humano mediado a través del lenguaje, es en sí misma, la escuela, ya que por su carácter formativo constituye el espacio donde convergen necesariamente el pensamiento, el lenguaje y el conocimiento, así como una fuerte y rica interacción social. Lo anterior nos hace reflexionar acerca de cuál metodología es la ideal y la que mejor se ajusta a las formas de comunicación que utilizamos para relacionarnos con el entorno físico y social, ya que el mismo lenguaje ejerce un papel insustituible en la percepción e interpretación del mundo y, sobre todo, en la forma de cómo aprendemos y el valor que le damos a lo aprendido, la invención y uso de nuevos códigos comunicativos y su influencia en el pensamiento.

Llegados a este punto, quizá no resulte extravagante afirmar que el lenguaje ha servido además como vehículo propio de una de las más curiosas actividades humanas, una que si bien algunos podrían no considerar como propiamente cognitiva, sin duda alguna involucra funciones cognitivas. Me refiero al maravilloso fenómeno de la expresión y creación artística.

Las implicaciones directas de esta afirmación nos sitúan ante un vasto panorama de nuevas posibilidades que podrían descifrar la infinidad de procesos mentales que el lenguaje lleva a cabo antes, durante y después del aprendizaje. A partir de esta idea, resulta indispensable dedicar una mirada a la formación y actitudes de los docentes y las docentes, a la metodología para el lenguaje y del lenguaje, que utilizarán y de las distintas formas de expresión e interacción posible que podrían generarse en cualquier contexto educativo.

No podemos negar que obedecemos a estímulos mayormente comunicativos, debido a nuestra condición humana. Esto nos lleva a cuestionar; cuáles son estos estímulos y de qué forma intervienen en el lenguaje y el aprendizaje, así como la génesis de conocimiento personal de cada individuo y, consecuentemente, en las metodologías que deben atender integral y eficazmente tal diversidad.

Se hace necesario, por lo tanto, dirigir la atención a la generación de nuevas teorías que conduzcan a novedosas concepciones del diseño curricular, la enseñanza del lenguaje como herramienta para el aprendizaje y el arte de la producción textual, muy diferentes de las que comúnmente se encuentran, en la actualidad, en las instituciones educativas. Igualmente importante es comprender y asimilar que el incremento de investigaciones interdisciplinarias y nuevos aportes científicos que, desde la neurociencia, han comenzado a hacer, de cierta manera, más visible el camino que conduce a la revaloración del lenguaje como itinerario para el fortalecimiento y pertinencia de la práctica pedagógica en la actual coyuntura educativa. 
En el ámbito educativo este proceso se expresa en la configuración de un nuevo paradigma que nos obliga a concebir el lenguaje como el proceso mediante el cual cada persona construye la plataforma perceptiva y comunicativa necesaria para apropiarse de los conocimientos, las habilidades, las destrezas y los valores que requiere para su desarrollo integral como ser humano. Dentro de esta visión, el leguaje debe concebirse como una herramienta innata de cada persona para lograr su plenitud expresiva.

En este nuevo paradigma educativo, cada persona es co y autoconstructora de su propio conocimiento más que consumidora del mismo. Por lo tanto, la acción docente se debe reconfigurar en la de guía del estudiante en el proceso de optimización del lenguaje, herramienta indispensable para construir y procesar conocimiento. En esta génesis del conocimiento inicial mediado por el lenguaje, el mismo es enriquecido o transformado significativamente durante el proceso, al aprender vocabulario, codificar nuevos significados, establecer relaciones semánticas y estimular la producción de ideas. Esta perspectiva del conocimiento está en contraposición con la visión más tradicional del mismo, consistente principalmente en un proceso de transmisión o adquisición de «parcelas» o «trozos» de conocimiento, conducente a una concepción errada y simplificada de procesos altamente complejos.

Desde una renovada visión de los formadores y formadoras, se hace impostergable una detallada revisión de nuestros supuestos epistemológicos, lingüísticos y ontológicos, así como sus implicaciones en el orden educativo. Al respecto, los centros de formación de docentes requieren homogenizar y uniformar el discurso pedagógico en torno al tema del lenguaje, la comunicación y la expresión, para definir los parámetros mínimos que deben ser entendidos, no solo en el ínterin universitario, sino, además, con una fuerte intervención en el sistema formal de enseñanza.

Finalmente, el tema deja una tarea para que en todos los escenarios educativos se trabaje por una renovada visión acerca del poder del lenguaje en torno a cómo percibimos el mundo, la realidad $\mathrm{y}$, por consecuencia, el lenguaje como detonador y conductor de ideas, en una relación equitativa entre la sociedad actual y futura. Abriendo brechas que permitan a las futuras generaciones gozar del lenguaje pleno e inherente de los seres humanos y como mecanismo para la expresión y la libertad de todas y todos (...)

"Sólo hay mundo donde hay lenguaje"

Martin Heidegger

\section{Referencias bibliográficas}

Arellano, S. (2002). La lectura comprensiva en el currículo escolar. Educación Primaria y Educación Secundaria Obligatoria. España: Gobierno de Navarra. Departamento de Educación y Cultura. Recuperado de http://www.pnte.cfnavarra.es/bibliotecasescolares/ blitz files/Blitzamar1\%20cas.pdf

Ellis, J. M. (1994). Language, Thought and Logic (Rethinking Theory) [Lenguaje, pensamiento y lógica (Repensar la teoría)]. Evanston, Illinois, United States: Northwestern University Press. 
Goodman K. (2008). El lenguaje integral: un camino fácil para el desarrollo del lenguaje. En M. I. Borrero (Comp.), Lecturas complementarias para los maestros. Leer y escribir con los niños (pp. 107-126). Colombia: Fundación Corona. Recuperado de http://www.fcorona.org/ descargas/publicaciones/educacion/EDU 47 LeerEscribir.pdf\#page =108

Olson, D. (1999). El mundo sobre el papel. El impacto de la escritura y la lectura en la estructura del conocimiento. España: Gedisa.

Ratey, J. (2003). El cerebro: manual de instrucciones. Barcelona: Random House. 